\title{
Solving elliptic diophantine equations by estimating linear forms in elliptic logarithms
}

\author{
by \\ R. J. Strokker (Rotterdam) and N. Tzanakis (Iraklion)
}

1. Introduction. In [Z], Zagier describes several methods for explicitly computing (large) integral points on models of elliptic curves defined over $\mathbb{Q}$. Here we are interested in the computation of all integral points on a given Weierstraß equation for an elliptic curve $E / \mathbb{Q}$, but not merely by reducing the original diophantine equation to an equivalent finite set of Thue equations which are subsequently solved by elementary, algebraic or analytic methods (see $[\mathrm{TdW}]$ and $[\mathrm{STz}]$ ). On the contrary, we adopt a more natural approach, one in which the linear (group) relation between an integral point and the generators of the free component of the Mordell-Weil group is directly transformed into a linear form in elliptic logarithms. This idea is not new; see [Ma, App. IV], [La, Ch. VI, §8], and [S1, Ch. IX, §5]. To make it work, that is to say, in order to produce upper bounds for the coefficients in the original linear (group) relation, one needs an effective lower bound for the linear form in elliptic logarithms. First to obtain such lower bounds were D. W. Masser [Ma, App. IV], in the case of elliptic curves with complex multiplication, and G. Wüstholz [Wu]; see also the bibliography in $[\mathrm{H}]$. We felt that the recent result of N. Hirata-Kohno [H, Coroll. 2.16] should serve our purpose best. Unfortunately, this result, being rather more general than we required, though effective, is not completely explicit. At our request, S. David kindly undertook the highly non-trivial task of making explicit the special case we needed. It is S. David's result [D, Th. 2.1] that is applied here for the first time to provide explicit upper bounds for the coefficients in the linear (group) relation corresponding to a given Weierstraß equation. We shall show by example that these bounds may be reduced to manageable proportions.

In the following sections we shall give a detailed description of the method referred to above. In the final section we present two examples, worked out in detail, of elliptic curves taken from the literature. Our choice 
seems rather arbitrary, but both examples are of some interest as illustrations of the method, and also in view of the difficulties one has to overcome when trying to solve the corresponding diophantine equations by traditional methods.

The equations referred to above are

$$
6 y^{2}=(x+1)\left(x^{2}-x+6\right),
$$

and

$$
y^{2}=(x+337)\left(x^{2}+337^{2}\right) .
$$

The corresponding elliptic curves have rank 2 and 3 respectively.

2. Preliminaries. This section is devoted to the introduction of the necessary concepts and to setting up the relevant notation.

We are interested in computing explicitly all solutions $(X, Y) \in \mathbb{Z} \times \mathbb{Z}$ of the equation

$$
Y^{2}+a_{1} X Y+a_{3} Y=X^{3}+a_{2} X^{2}+a_{4} X+a_{6},
$$

where $a_{1}, a_{2}, a_{3}, a_{4}, a_{6}$ are rational integers. This equation defines an elliptic curve $E / \mathbb{Q}$, provided it has a non-vanishing discriminant. From now on we assume that this is the case.

A linear transformation

$$
X=u^{2} x+v, \quad Y=u^{3} y+w u^{2} x+z
$$

for suitably chosen $u, v, w, z \in \mathbb{Q}, u \neq 0$, gives another equation for $E$ of the form

$$
y^{2}=f(x),
$$

where $f \in \mathbb{Q}[x]$ is a cubic polynomial

$$
f(x)=x^{3}+a x+b
$$

with non-zero discriminant. The latter equation (3) is often more convenient.

Throughout this paper, an integral point will always be a point $P=$ $(X(P), Y(P))$ with rational integral coordinates satisfying (1); the possibly non-integral coordinates of the point $P$ on the corresponding equation (3), will be denoted by $(x(P), y(P))$.

Let $r$ be the rank of the Mordell-Weil group $E(\mathbb{Q})$. We assume $r \geq 1$ as the case $r=0$ is rather trivial. By the Mordell-Weil theorem we have the following group isomorphism

$$
E(\mathbb{Q}) \cong E_{\text {tors }}(\mathbb{Q}) \times \mathbb{Z}^{r} .
$$

The set of generators of $E(\mathbb{Q}) / E_{\text {tors }}(\mathbb{Q})$ will be denoted by $\left\{P_{1}, \ldots, P_{r}\right\}$. We shall always tacitly assume that such a set can be explicitly determined. 
For any $P \in E(\mathbb{Q})$, there exist rational integers $m_{1}, \ldots, m_{r}$ such that

$$
P=m_{1} P_{1}+\ldots+m_{r} P_{r}+T
$$

for some $T \in E_{\text {tors }}(\mathbb{Q})$. The construction of the (finite) torsion group $E_{\text {tors }}(\mathbb{Q})$ should not pose any problems, so that $(4)$ can be seen as a direct link between the (unknown) point $P$ and the integral vector $\mathbf{m}=$ $\left(m_{1}, \ldots, m_{r}\right)$. For integral $P$ we would like to estimate the vector $\mathbf{m}$. This should be possible, at least in principle, as the number of such points $P$ is finite. In other words, if the integral point $P=(X(P), Y(P))$ satisfies (1), then $|X(P)|$ and $|Y(P)|$ are bounded, which means that $P$ cannot be too close to the identity $O$ of the group $E(\mathbb{Q})$.

In order to use the information provided by (4) numerically, we need a real valued function that does not disturb the linear character of (4), that maps the identity $O$ to 0 and measures in some sense the distance from $O$. Now, the group $E(\mathbb{R})$ is isomorphic to one or two copies of the circle group $\mathbb{R} / \mathbb{Z}$, depending on the number of real zeros of $f(x)$. Let $\gamma$ be the largest (possibly the only) real root of $f(x)=0$. As the integral points $P$ satisfying $X(P)<u^{2} \gamma+v$ can be easily found by direct search, we will concentrate on those integral $P$ which belong to the component of $E(\mathbb{R})$ containing the identity $O$ (i.e. the infinite component), namely

$$
E_{0}(\mathbb{R})=\left\{P \in E \cap \mathbb{R}^{2} \mid x(P) \geq \gamma\right\} \cup\{O\} .
$$

The group isomorphism

$$
\phi: E_{0}(\mathbb{R}) \rightarrow \mathbb{R} / \mathbb{Z}
$$

can be given explicitly as follows. Let

$$
\omega=2 \int_{\gamma}^{\infty} \frac{d t}{\sqrt{f(t)}} .
$$

This is the fundamental real period of the Weierstra $§ \wp$-function associated with the curve given by (3). For $P \in E_{0}(\mathbb{R})$ (see [Z, p. 429]),

$$
\phi(P) \equiv \begin{cases}0(\bmod 1) & \text { if } P=O, \\ \frac{1}{\omega} \int_{x(P)}^{\infty} \frac{d t}{\sqrt{f(t)}}(\bmod 1) & \text { if } y(P) \geq 0, \\ -\phi(-P)(\bmod 1) & \text { if } y(P) \leq 0 .\end{cases}
$$

Clearly, there is no loss of generality in assuming that $\phi(P) \in[0,1)$.

It is our goal to establish an effectively computable upper bound for $|\phi(P)|$ depending on the coefficients $m_{1}, \ldots, m_{r}$ only. Because of (4) this results in an upper bound for a linear form in $\phi\left(P_{1}\right), \ldots, \phi\left(P_{r}\right)$, essentially the elliptic logarithms. Combining this upper bound with David's lower bound 
for linear forms in elliptic logarithms (see [D, Th. 2.1] and the Appendix) clinches the argument in so far as an effectively computable upper bound for $\max _{1 \leq i \leq r}\left|m_{i}\right|$ is obtained in this way.

In the course of finding the upper bound for $|\phi(P)|$ we mentioned above, we need the canonical or Néron-Tate height function $\widehat{h}$. This function is a positive definite quadratic form on $E(\mathbb{Q}) / E_{\text {tors }}(\mathbb{Q})$. To be more precise-we follow Silverman, see [S1, Ch. VIII, §9])—if

$$
\langle P, Q\rangle=\widehat{h}(P+Q)-\widehat{h}(P)-\widehat{h}(Q)
$$

is the so-called Néron-Tate (or Weil) pairing, and $P$ is expressed as in (4), then

$$
\widehat{h}(P)=\frac{1}{2} \sum_{1 \leq i, j \leq r}\left\langle P_{i}, P_{j}\right\rangle m_{i} m_{j}
$$

and the matrix $\mathcal{H}=\left(\frac{1}{2}\left\langle P_{i}, P_{j}\right\rangle\right)_{r \times r}$ is positive definite (see [S1, Prop. 9.6, p. 232]). Relation (6) immediately follows from the facts that for any $P \in$ $E(\overline{\mathbb{Q}})$ and any $m \in \mathbb{Z}$,

- the Néron-Tate pairing is bilinear,

- $\widehat{h}(m P)=m^{2} \widehat{h}(P)$,

- $\widehat{h}(P) \geq 0$ and $\widehat{h}(P)=0$ if and only if $P$ is a torsion point.

3. Basic inequalities. In this section we shall establish a few elementary inequalities that are crucial in the derivation of our upper bound for $|\phi(P)|$. In each inequality we introduce an absolute constant accordingly labeled.

InEQuAlity 1. Let $P \in E(\mathbb{Q})$ be expressed as in (4). Then

$$
\widehat{h}(P) \geq c_{1} \max _{1 \leq i \leq r} m_{i}^{2},
$$

where $c_{1}$ is the least eigenvalue of the positive definite matrix $\mathcal{H}$ introduced in (6).

Pr o of. According to (6) we have

$$
\widehat{h}(P)=\mathbf{m}^{\mathrm{T}} \mathcal{H} \mathbf{m},
$$

where $\mathbf{m}$ is the column vector with components $m_{1}, \ldots, m_{r}$. As $\mathcal{H}$ is symmetric, a diagonal matrix $\Lambda$ of eigenvalues of $\mathcal{H}$ and an orthogonal matrix $\mathcal{Q}$ exist such that $\mathcal{H}=\mathcal{Q}^{\mathrm{T}} \Lambda \mathcal{Q}$. Writing $\mathbf{n}=\mathcal{Q} \mathbf{m}$ and observing that $\mathcal{Q}^{\mathrm{T}} \mathcal{Q}=I$, 
we deduce

$$
\begin{aligned}
\widehat{h}(P) & =\mathbf{m}^{\mathrm{T}} \mathcal{Q}^{\mathrm{T}} \Lambda \mathcal{Q} \mathbf{m}=\mathbf{n}^{\mathrm{T}} \Lambda \mathbf{n}=\sum_{i=1}^{r} \lambda_{i} n_{i}^{2} \\
& \geq c_{1} \sum_{i=1}^{r} n_{i}^{2}=c_{1} \mathbf{n}^{\mathrm{T}} \mathbf{n}=c_{1} \mathbf{m}^{\mathrm{T}} \mathcal{Q}^{\mathrm{T}} \mathcal{Q} \mathbf{m}=c_{1} \mathbf{m}^{\mathrm{T}} \mathbf{m} \\
& =c_{1} \sum_{i=1}^{r} m_{i}^{2} \geq c_{1} \max _{1 \leq i \leq r} m_{i}^{2}
\end{aligned}
$$

as claimed.

INEQUALITY 2. Let $\gamma, \gamma^{\prime}, \gamma^{\prime \prime}$ be the roots of $f(x)=0$ and put $c_{2}=$ $2 \max \left\{|\gamma|,\left|\gamma^{\prime}\right|,\left|\gamma^{\prime \prime}\right|\right\}$. Then, for all $x \geq c_{2}$,

$$
\left|\int_{x}^{\infty} \frac{d t}{\sqrt{f(t)}}\right| \leq 4 \sqrt{2}|x|^{-1 / 2}
$$

Pro o f. For $t \geq x \geq c_{2}$ we have $0<f(t)=t^{3}+a t+b=|t-\gamma|\left|t-\gamma^{\prime}\right|\left|t-\gamma^{\prime \prime}\right|$ and as $t$ is larger than the absolute largest zero of $f(x)$, it follows that $|t-\gamma| \geq t-|\gamma| \geq t / 2$, and likewise for $\gamma^{\prime}$ and $\gamma^{\prime \prime}$. Consequently, $1 / \sqrt{f(t)} \leq$ $2^{3 / 2} t^{-3 / 2}$ and hence, for all $N>x$,

$$
\int_{x}^{N} \frac{d t}{\sqrt{f(t)}} \leq \int_{x}^{N} 2^{3 / 2} t^{-3 / 2} d t=4 \sqrt{2}\left(x^{-1 / 2}-N^{-1 / 2}\right) .
$$

Letting $N$ tend to infinity completes the proof.

Before proceeding, let us remind the reader that there is another, in some sense more natural height function than the canonical height $\widehat{h}$. For any rational number $\varrho=m / n$ with $\operatorname{gcd}(m, n)=1$,

$$
h(\varrho):=\log \max \{|m|,|n|\}
$$

is known as the absolute logarithmic height of $\varrho$. Now the naive height of a point $P \in E(\mathbb{Q}), P \neq O$, is defined as the absolute logarithmic height of $X(P)$.

INEQUality 3. Let $u, v$ and $\gamma$ be as in Section 2. Let $X_{0}$ be a positive integer strictly larger than $v$. Put

$$
\begin{aligned}
& c_{0}= \begin{cases}\log |u| & \text { if } v \leq 0, \\
\log |u|+\frac{1}{2} v\left(X_{0}-v\right)^{-1} & \text { if } v>0,\end{cases} \\
& c_{3}=c_{0}+\frac{1}{12} \log |\Delta|+\frac{1}{12} \log ^{+}|j|+\frac{1}{2} \log ^{+}\left|b_{2} / 12\right|+\frac{1}{2} \log 2^{*}+1.07,
\end{aligned}
$$

where $\Delta$ and $j$ are the discriminant and the $j$-invariant of the elliptic curve $E / \mathbb{Q}$ defined by (1), $\log ^{+}|\alpha|:=\log \max \{1,|\alpha|\}$ for $\alpha \in \mathbb{R}, b_{2}=a_{1}^{2}+4 a_{2}$ and $2^{*}=1$ or 2 according as $b_{2}$ vanishes or not, respectively. 
Then, for all $P \in E(\mathbb{Q})$ with $X(P) \geq X_{0}$, we have $x(P)>0$, and

$$
\widehat{h}(P)-\frac{1}{2} \log x(P) \leq c_{3} .
$$

Remark. There is no restriction on the choice of model to which we apply Silverman's theorem [S3, Th. 1.1], so long as $a_{1}, \ldots, a_{6} \in \mathbb{Z}$. Therefore, we may choose a model that provides a small constant $c_{3}$, subject to $a_{1}, \ldots, a_{6} \in \mathbb{Z}$. A natural choice is the global minimal Weierstraß model, or the model that minimizes $\log ^{+}\left|b_{2} / 12\right|$.

Proof of Inequality 3 . Clearly $x(P)=u^{-2}(X(P)-v)>0$. Next, by [S3, Th. 1.1] we have

$$
\widehat{h}(P)-\frac{1}{2} h(X(P)) \leq c_{3}-c_{0}
$$

and, since $P$ is an integral point, $h(X(P))=\log X(P)$. Therefore

(8) $h(X(P))=\log \left(u^{2} x(P)+v\right)=2 \log |u|+\log x(P)+\log \left(1+\frac{v}{u^{2} x(P)}\right)$.

If $v \leq 0$ then the final logarithm in (8) is non-positive, and for positive $v$,

$$
\log \left(1+\frac{v}{u^{2} x(P)}\right)<\frac{v}{u^{2} x(P)}=\frac{v}{X(P)-v} \leq \frac{v}{X_{0}-v} .
$$

Combining this with (7) and (8) completes the proof.

4. The linear form in elliptic logarithms. As we are interested in finding all integral points on (1), and as points with small $X$-coordinate can be found without fail by direct search, we focus our attention on points $P \in E_{0}(\mathbb{Q}), P \neq O$ with $X(P) \geq X_{0}$, for some conveniently chosen positive integer $X_{0}$. Elaborating on this choice of $X_{0}$, we first point out that a point $P$ of $E(\mathbb{R})$ belongs to $E_{0}(\mathbb{R})$ if and only if $x(P) \geq \gamma$ or, equivalently, if and only if $X(P) \geq u^{2} \gamma+v$. In view of this and the requirements set down in Inequalities 2 and 3 , we take $X_{0}=\left\lfloor\max \left\{c_{2}, u^{2} \gamma+v, v\right\}\right\rfloor+1$.

Let $P$ be expressed in terms of the generators of the free component of $E(\mathbb{Q})$ as in (4). We put

$$
M=\max _{1 \leq i \leq r}\left|m_{i}\right| .
$$

Applying the isomorphism $\phi$ to (4) - but see the remark below-yields

$$
\phi(P) \equiv m_{1} \phi\left(P_{1}\right)+\ldots+m_{r} \phi\left(P_{r}\right)+\phi(T)(\bmod 1),
$$

and hence an integer $m_{0}$ exists such that

$$
\phi(P)=m_{0}+m_{1} \phi\left(P_{1}\right)+\ldots+m_{r} \phi\left(P_{r}\right)+\phi(T),
$$

so that, assuming all $\phi$-values belong to $[0,1)$,

$$
\left|m_{0}\right|<\left|m_{1}\right|+\ldots+\left|m_{r}\right|+1 \leq r M+1 \text {. }
$$

For our purpose, it clearly suffices to compute an upper bound for $M$. 
Re mark. A problem arises when some or all of the generators $P_{i}$ do not belong to $E_{0}(\mathbb{Q})$. This is only possible when $f(x)=0$ has three real roots $\gamma>\gamma^{\prime}>\gamma^{\prime \prime}$. Then $R:=\left(\gamma^{\prime}, 0\right)$ is a point of order two with real algebraic coordinates. Now, for each $i=1, \ldots, r$, if $P_{i} \notin E_{0}(\mathbb{Q})$ then replace $P_{i}$ in (4) by $P_{i}+R$-which does belong to $E_{0}(\mathbb{R})$ - and adjust $T$ accordingly.

Let $t$ be the order of the torsion point $T$. Then, $t \phi(T) \equiv \phi(O) \equiv 0$ $(\bmod 1)$, and hence $\phi(T)=s / t$, for some non-negative integer $s<t$. Thus,

$$
\phi(P)=\left(m_{0}+\frac{s}{t}\right)+m_{1} \phi\left(P_{1}\right)+\ldots+m_{r} \phi\left(P_{r}\right) .
$$

On the other hand, by Inequalities 1 and 3,

$$
\log x(P) \geq 2\left(\widehat{h}(P)-c_{3}\right)>2\left(c_{1} M^{2}-c_{3}\right) .
$$

Therefore, $|x(P)|^{-1 / 2}=(x(P))^{-1 / 2} \leq \exp \left(c_{3}-c_{1} M^{2}\right)$. In view of Inequality 2 and the definition of $\phi$, it follows that

$$
|\phi(P)|=\left|\frac{1}{\omega} \int_{x(P)}^{\infty} \frac{d t}{\sqrt{f(t)}}\right| \leq \frac{4 \sqrt{2}}{\omega}|x(P)|^{-1 / 2} \leq \frac{4 \sqrt{2}}{\omega} \exp \left(c_{3}-c_{1} M^{2}\right) .
$$

On writing

$$
L(P):=\omega \phi(P)=\left(m_{0}+\frac{s}{t}\right) \omega+m_{1} u_{1}+\ldots+m_{r} u_{r},
$$

where $u_{i}=\omega \phi\left(P_{i}\right)$ for $i=1, \ldots, r$, we see that (12) induces the upper bound for the linear form $L(P)$ in elliptic logarithms hinted at above. Indeed, if we denote by $\wp$ the Weierstraß $\wp$-function, which parameterizes $E$, then

$$
\wp\left(u_{i}\right)=\wp\left(\int_{x(P)}^{\infty} \frac{d t}{\sqrt{f(t)}}\right)=x(P), \quad i=1, \ldots, r
$$

(see for instance [WW, Ch. XX, no 20.221]). Note that $\omega$ is a pole of $\wp$. As the linear form $L(P)$ is non-vanishing, because $P \neq O$ implies $\phi(P) \neq 0$, we may apply S. David's theorem (see the Appendix) to obtain the lower bound

$$
|L(P)|>\exp \left(-c_{4}\left(\log M^{\prime}+c_{5}\right)\left(\log \log M^{\prime}+c_{6}\right)^{r+2}\right)
$$

for explicitly computable positive constants $c_{4}, c_{5}$ and $c_{6}$, where

$$
\log M^{\prime}:=\max \left\{\log M, h\left(m_{0}+s / t\right)\right\},
$$

provided that $M$ is not less than some explicitly computable constant $M_{0} \geq$ 16. Combining upper and lower bounds (12) and (14) then yields

$$
c_{1} M^{2}<c_{4}\left(\log M^{\prime}+c_{5}\right)\left(\log \log M^{\prime}+c_{6}\right)^{r+2}+c_{3}+\log (4 \sqrt{2}) .
$$


By (10) and the definition of $M^{\prime}$, we have

$$
\begin{aligned}
M^{\prime} \leq\left|m_{0} t+s\right|<t_{0}(r M+1)+t_{0}-1, & \\
t_{0} & :=\max \left\{\operatorname{order}(T) \mid T \in E_{\text {tors }}(\mathbb{Q})\right\},
\end{aligned}
$$

where $t_{0} \leq 12$ by Mazur's theorem (see [S1, p. 223]). From this inequality we easily deduce that

$$
\log M^{\prime}<\log M+\log \left(t_{0} r\right)+\frac{2 t_{0}-1}{16 t_{0} r}
$$

so that (15) implies

\section{PRINCIPAL INEQUALITY.}

(16) $M^{2}<c_{3} c_{1}^{-1}+c_{1}^{-1} \log (4 \sqrt{2})+c_{4} c_{1}^{-1}\left(\log M+c_{7}\right)\left(\log \log M+c_{8}\right)^{r+2}$,

where

$c_{7}=c_{5}+\log \left(t_{0} r\right)+\frac{2 t_{0}-1}{16 t_{0} r} \quad$ and $\quad c_{8}=c_{6}+\left(\log \left(t_{0} r\right)+\frac{2 t_{0}-1}{16 t_{0} r}\right) / \log 16$.

Now clearly (16) provides an effectively computable upper bound for $M$.

5. Reduction of the upper bound. Inequalities (12) and (16) may be rewritten in simplified form as

$$
|\phi(P)|<K_{1} \exp \left(-K_{2} M^{2}\right) \text { and } M<K_{3},
$$

where $K_{1}, K_{2}, K_{3}$ are explicitly known and $K_{3}$ is "very large" -in the two numerical examples of Section 6 it is of magnitude $10^{38}$ and $10^{59}$, respectively. Since such a large upper bound for $M$ is way out of reach of any practical search method, we will try to reduce it.

Consider the $(r+1)$-dimensional lattice, generated by the columns of the matrix

$$
\mathcal{A}=\left(\begin{array}{cccc}
1 & \ldots & 0 & 0 \\
0 & \ldots & 0 & 0 \\
\vdots & \ddots & \vdots & \vdots \\
0 & \ldots & 1 & 0 \\
{\left[K_{0} \phi\left(P_{1}\right)\right]} & \ldots & {\left[K_{0} \phi\left(P_{r}\right)\right]} & K_{0}
\end{array}\right)
$$

Here $K_{0}$ is a conveniently chosen integer, larger than $K_{3}^{r+1}$ - this choice of $K_{0}$ will be further discussed in the lines following the Proposition. Further, [.] means rounding towards 0 , i.e. $[\alpha]=\lceil\alpha\rceil$ if $\alpha \leq 0$, and $[\alpha]=\lfloor\alpha\rfloor$ if $\alpha>0$. 
Let $\left(m_{1}, \ldots, m_{r}, m_{0}\right) \in \mathbb{Z}^{r+1}$ satisfy $\left|m_{i}\right|<K_{3}$ for $i=0,1, \ldots, r$, and consider the lattice point

$$
\boldsymbol{\ell}=\mathcal{A}\left(\begin{array}{c}
t m_{1} \\
\vdots \\
t m_{r} \\
t m_{0}+s
\end{array}\right)=\left(\begin{array}{c}
t m_{1} \\
\vdots \\
t m_{r} \\
\lambda
\end{array}\right)
$$

where

$$
\lambda:=t m_{1}\left[K_{0} \phi\left(P_{1}\right)\right]+\ldots+t m_{r}\left[K_{0} \phi\left(P_{r}\right)\right]+\left(t m_{0}+s\right) K_{0} .
$$

Since $\left|\lambda-K_{0} t \phi(P)\right| \leq r t M \leq r t K_{3}$-recall (11) -it follows that

$$
\|\ell\|^{2}=t^{2}\left(m_{1}^{2}+\ldots+m_{r}^{2}\right)+\lambda^{2} \leq r t^{2} K_{3}^{2}+t^{2}\left(K_{0}|\phi(P)|+r K_{3}\right)^{2} .
$$

On the other hand, if the lattice basis $\left\{\mathbf{b}_{1}, \ldots, \mathbf{b}_{r+1}\right\}$ is reduced in the sense of [LLL], we have

$$
\left\|\mathbf{b}_{1}\right\|^{2} \leq 2^{r}\|\ell\|^{2}
$$

in view of Proposition (1.11) of the paper cited. Combining this with (19) yields

$$
K_{0}|\phi(P)| \geq \sqrt{t^{-2} 2^{-r}\left\|\mathbf{b}_{1}\right\|^{2}-r K_{3}^{2}}-r K_{3},
$$

which gives, after applying the first inequality of (17),

$$
M^{2} \leq K_{2}^{-1}\left(\log \left(K_{0} K_{1}\right)-\log \left(\sqrt{t^{-2} 2^{-r}\left\|\mathbf{b}_{1}\right\|^{2}-r K_{3}^{2}}-r K_{3}\right)\right),
$$

provided the right-hand side of (20) is positive, i.e.

$$
\left\|\mathbf{b}_{1}\right\|>2^{r / 2} t K_{3} \sqrt{r^{2}+r} .
$$

We have thus proven the following

Proposition. If the first vector $\mathbf{b}_{1}$ of an $L L L$-reduced basis for the lattice generated by the column vectors of the matrix $\mathcal{A}$ in (18) satisfies (22), then an upper bound for $M$ is given by (21).

Heuristically, it can be argued (see for instance [TdW, Sect. 3.1]) that the length of $\mathbf{b}_{1}$ is of the same magnitude as $K_{0}^{1 /(r+1)}$. Therefore, if we choose $K_{0}$ to be somewhat larger than $\left(2^{r / 2} t K_{3} \sqrt{r^{2}+r}\right)^{r+1}$, it is reasonable to expect that (22) is satisfied - if not, we choose a larger $K_{0}$ - so that the Proposition can be applied. Note that the initial bound $K_{3}$ of $M$ is thus reduced to a new bound which is of the size of $\sqrt{\log K_{3}}$. If the reduced bound is not small enough, then we repeat the above procedure with the reduced bound replacing $K_{3}$.

For the computation of an LLL-reduced basis we have applied de Weger's version of the LLL-algorithm. For a detailed description the reader should consult de Weger's book [dW, Ch. 3]. 
6. Applications. In this section we shall apply the method described above to the two equations mentioned in the introduction.

The first application concerns the determination of all integer points on Mordell's diophantine equation

$$
6 Y^{2}=(X+1)\left(X^{2}-X+6\right) .
$$

In his book [Mo, p. 259] Mordell asks whether all integer solutions of this equation are given by $X=-1,0,2,7,15,74$. W. Ljunggren answered Mordell's question in [Lj] by adding one more point. Subsequently, Andrew Bremner gave a simpler proof in [Br]. Both methods are ingenious but complicated and their applicability to the general case appears to be limited. With our method the solution process is rather straightforward. We shall confirm that

EXAMPLE 1. The only integer solutions $(X, Y)$ with $Y \geq 0$ of (23) are

$$
(-1,0),(0,1),(2,2),(7,8),(15,24),(74,260),(767,8672) \text {. }
$$

In order to live up to this claim we have to construct a set of generators of infinite order for $E(\mathbb{Q})$, and the relevant constants have to be calculated. First we transform (23) to Weierstraß form. This gives

$$
y^{2}=x^{3}+180 x+1296 .
$$

The rank of the corresponding curve is 2; in fact Ian Connell's Apecs 2.7 gives this (complete) set of independent points of infinite order on (24): $\{(-3,27),(10,64)\}$. Further, the torsion subgroup $E_{\text {tors }}(\mathbb{Q})$ is of order two and its generator is $T=(-6,0)$. It is not hard to show that the points $P_{1}=$ $(-3,27)$ and $P_{2}=(10,64)$ generate the Mordell-Weil group modulo torsion. For, the set $\mathcal{S}$ of 8 points $O, T, P_{1}, P_{1}+T, P_{2}, P_{2}+T, P_{1}-P_{2}, P_{1}-P_{2}+T$ represents $E(\mathbb{Q}) / 2 E(\mathbb{Q})$. Indeed, it is easily checked - again, Apecs is useful here, or one may use reduction modulo a few small primes - that none of these points, with the obvious exception of $O$, can be written as twice a point of $E(\mathbb{Q})$. Now by [S3, Prop. 7.2] the set of points $P$ with

$$
\widehat{h}(P) \leq \max \{\widehat{h}(X) \mid X \in \mathcal{S}\} \leq 0.8787
$$

generates $E(\mathbb{Q}) / E_{\text {tors }}(\mathbb{Q})$.

In order to set up a search for these points, we compare the canonical height with the naive height. Applying [S3, Th. 1.1] here yields

$$
\frac{1}{2} h(X(P)) \leq \widehat{h}(P)+\frac{1}{8} h(j)+\frac{1}{12} h(\Delta)+0.973,
$$

and, as $j_{E}=13500 / 23$ and $\Delta=-2^{16} 3^{6} 23$, this amounts to searching for all points $X(P)=R / S^{2}$ with $\max \left\{|R|, S^{2}\right\}<3705$. We used Upecs-the little brother of Apecs, written in the very fast UBASIC 8.21 by Y. Kida-to perform this search, and as it happened, all points uncovered were linear combinations of $P_{1}, P_{2}$, and $T$. 
In accordance with the definitions and notations as laid down in the Appendix at the end of this paper, we have $a=2^{2} 3^{2} 5, b=2^{4} 3^{4}$, and hence

$$
\max \left\{1,|a / 4|_{p},|b / 16|_{p}\right\}= \begin{cases}3^{4} & \text { if } p=\infty, \\ 1 & \text { otherwise }\end{cases}
$$

so that $h(a / 4, b / 16) \approx 4.3944492$. Also $h\left(j_{E}\right)=\log (13500) \approx 9.5104450$, and therefore

$$
h_{E} \approx 9.5104450 .
$$

Also $\gamma=-6$ and the formulas (32) give

$$
\begin{gathered}
\omega_{1} \approx 1.0606085+0.81447364 i, \quad \omega_{2}=\bar{\omega}_{1}, \\
\tau=\frac{\omega_{1}}{\omega_{2}} \approx 0.25808531+0.96612213 i,
\end{gathered}
$$

and the fundamental real period for the Weierstraß $\wp$-function associated with $(24)$ is

$$
\omega=2 \Re \omega_{1} \approx 2.1212170 .
$$

Here we consider the following linear form in elliptic logarithms (see (13)):

$$
L(P)=\left(m_{0}+\frac{s}{2}\right) \omega+m_{1} u_{1}+m_{2} u_{2} .
$$

In the notation of David's Theorem (see the Appendix) we have $r=2$, $R_{i}=P_{i}(i=1,2)$, and $R_{0}=O$. Also $u_{i}=\omega \phi\left(P_{i}\right)(i=1,2)$, and $u_{0}=\omega$. Zagier's algorithm for the evaluation of the $\phi$-values [Z, (10) on p. 430]) gives

$$
\phi\left(P_{1}\right) \approx 0.40084555, \quad \phi\left(P_{2}\right) \approx 0.25694930,
$$

and hence, by the definition of the $u_{i}$ 's, it follows that

$$
u_{1} \approx 0.85028037, \quad u_{2} \approx 0.54504522 .
$$

Using Apecs - the algorithm in [S2] could also be used - we computed

$$
\widehat{h}\left(P_{1}\right) \approx 0.87867020, \quad \widehat{h}\left(P_{2}\right) \approx 0.70055495,
$$

and of course, $\widehat{h}\left(R_{0}\right)=0$.

Then, by the definition of the $A_{i}$ 's, we see that we can take

$$
A_{0}=24.55, \quad A_{1}=A_{2}=9.511, \quad \mathcal{E}=e .
$$

It follows that we may choose in (16)

$$
c_{4}=9.655 \cdot 10^{69} \text {. }
$$

Further, with $t_{0}=2, c_{5}=1, c_{6}=1+h_{E}$ in (16), we can also choose

$$
c_{7}=2.434, \quad c_{8}=11.03 .
$$

In (2) we take

$$
u=1, \quad v=w=z=0,
$$


so that, in particular, $X(P)=x(P)$. Using MapleV and Apecs we computed the least eigenvalue of the matrix $\mathcal{H}$ introduced in (6), and we found (see Inequality 1)

$$
c_{1} \approx 0.26833321 \text {. }
$$

We choose $c_{1}=0.2683$ in (16). Moreover, we have

$$
\gamma=-6, \quad \gamma^{\prime}=3+3 \sqrt{23} i, \quad \gamma^{\prime \prime}=\overline{\gamma^{\prime}},
$$

which gives, by Inequality 2 ,

$$
c_{2}=12 \sqrt{6} .
$$

In the first paragraph of Section 4 , it was observed that $X_{0}$ may be chosen as $\left\lfloor\max \left\{c_{2}, u^{2} \gamma+v, v\right\}\right\rfloor+1=30$, so that our search concerns all points $P$ with $X(P) \geq 30$. It is straightforward to check, even by hand, that the only integral points $(x, y)$ on $(24)$ with $x<30$ are

$$
(-6,0),(0,36),(12,72) \text {. }
$$

By Inequality $3, c_{3} \approx 3.3360395$, so we can take $c_{3}=3.337$ and (16) now yields

$$
M<4.368 \cdot 10^{38} .
$$

Next we apply the reduction process of Section 5 to the relevant linear form $\phi(P)$ (see (11)). In view of (12) we may take

$$
K_{1}=75.03>\frac{4 \sqrt{2}}{\omega} \exp \left(c_{3}\right), \quad K_{2}=0.2683,
$$

and, because of the upper bound for $M$,

$$
K_{3}=4.368 \cdot 10^{38} \text {. }
$$

Further, we choose

$$
K_{0}=10^{120},
$$

which is somewhat larger than $\left(4 \sqrt{6} K_{3}\right)^{3}$.

In view of (18), this choice of $K_{0}$ forces us to compute $\phi\left(P_{i}\right)$ for $i=1,2$ with a precision of 120 decimal digits. This is accomplished by executing Zagier's algorithm ([Z, (10) on p. 430] coded in UBASIC, which allows for very large precision. Finally, application of the LLL-algorithm - we used de Weger's version [dW, Sect. 3.5] and checked the result with the lllint procedure which is part of GP/PARI 1.37.3 - gives a reduced basis $\left\{\mathbf{b}_{\mathbf{1}}, \mathbf{b}_{\mathbf{2}}, \mathbf{b}_{\mathbf{3}}\right\}$ with

$$
\mathbf{b}_{\mathbf{1}}=\left(-6.476 \ldots \times 10^{39},-4.976 \ldots \times 10^{39}, 3.834 \ldots \times 10^{39}\right),
$$

from which we see that the inequality (22) with $r=t=2$ is satisfied. Hence, by the Proposition of Section $5, M \leq 26$. Repeating the process with $K_{1}$, 
$K_{2}$ as above and $K_{3}=26, K_{0}=10^{8}$, the LLL-algorithm gives a reduced basis with

$$
\mathbf{b}_{\mathbf{1}}=(-101,-146,266) \text {. }
$$

Thus, (22) is satisfied and the Proposition yields the new bound

$$
M \leq 8 .
$$

The result of a direct search - using Apecs again - for all integral points

$$
P=m_{1} P_{1}+m_{2} P_{2}+\varepsilon T, \quad 0 \leq m_{1} \leq 8, \quad\left|m_{2}\right| \leq 8, \quad \varepsilon \in\{0,1\},
$$

with $(x(P), y(P))$ on $(24)$ and $x(P) \geq 30$ is listed in the following table:

\begin{tabular}{ccc|cc}
\hline$m_{1}$ & $m_{2}$ & $\varepsilon$ & $x(P)$ & $y(P)$ \\
\hline 0 & -2 & 1 & 4602 & -312192 \\
0 & 2 & 1 & 4602 & 312192 \\
1 & -2 & 0 & 69 & -585 \\
1 & -1 & 0 & 42 & 288 \\
1 & 0 & 1 & 90 & -864 \\
2 & -1 & 1 & 444 & 9360 \\
\hline
\end{tabular}

From this we see that the only integral solutions $(x, y)$ of $(24)$ are those given by the $x(P)$-values in the table in addition to those given by $x=$ $-6,0,12$. Since the solutions $(X, Y)$ of $(23)$ and $(x, y)$ of $(24)$ are related by

$$
x=6 X, \quad y=36 Y,
$$

and as all $x$-values mentioned above, except 69 , are divisible by 6 , the integer solutions of (24) are as claimed in Example 1.

The next example deals with the Weierstraß equation

$$
Y^{2}=(X+337)\left(X^{2}+337^{2}\right) \text {. }
$$

We will show that

Example 2. The only integer solutions $(X, Y)$ with $Y \geq 0$ of (25) are

$$
(-337,0),(-287,3130),(2113,105910),(56784,13571615) .
$$

Necessary information on the characteristics of the elliptic curve given by $(25)$ can be found in $\left[\mathrm{STo}\right.$. In particular, $E_{\text {tors }}(\mathbb{Q}) \cong \mathbb{Z}_{2}$ with point of order two $T:=(-337,0)$ and set of generators $\left\{P_{1}, P_{2}, P_{3}\right\}$ of $E(\mathbb{Q}) / E_{\text {tors }}(\mathbb{Q})$, where

$$
\begin{gathered}
P_{1}=\left(\frac{5392}{9}, \frac{567845}{27}\right), \quad P_{2}=(56784,13571615), \\
P_{3}=\left(\frac{105144}{25}, \frac{35547097}{125}\right) .
\end{gathered}
$$


Further,

$$
a=\frac{2}{3} \cdot 337^{2}, \quad b=\frac{20}{27} \cdot 337^{3}, \quad j_{E}=128, \quad \text { and } \quad \Delta=-2^{8} 337^{6} .
$$

By (32) of the Appendix we computed the following pair of fundamental periods:

$$
\omega_{1} \approx 0.21988008+0.14965789 i, \quad \omega_{2}=\bar{\omega}_{1},
$$

and thus

$$
\tau=\frac{\omega_{1}}{\omega_{2}} \approx 0.36680841+0.93029651 i
$$

which satisfy the requirements of (30). It follows that

$$
\omega=2 \Re \omega_{1} \approx 0.43976016
$$

is a fundamental real period.

Next we computed $h(a / 4, b / 16)$. As

$$
\max \left\{1,|a / 4|_{p},|b / 16|_{p}\right\}= \begin{cases}2^{-2} 3^{-3} 5 \cdot 337^{3} & \text { if } p=\infty \\ 2^{2} & \text { if } p=2, \\ 3^{3} & \text { if } p=3, \\ 1 & \text { otherwise }\end{cases}
$$

we see that

$$
h_{E}=h\left(\frac{a}{4}, \frac{b}{16}\right) \approx 19.069687 .
$$

Here we consider the linear form in elliptic logarithms (see (13))

$$
L(P)=\left(m_{0}+\frac{s}{2}\right) \omega+m_{1} u_{1}+m_{2} u_{2}+m_{3} u_{3} .
$$

In the notation of David's Theorem (see the Appendix) we now have $r=3$, $R_{i}=P_{i}(i=1,2,3)$, and $R_{0}=O$. Also, $u_{i}=\omega \phi\left(P_{i}\right)$ for $i=1,2,3$, and $u_{0}=\omega$. Zagier's algorithm for the evaluation of the $\phi$-values $[\mathrm{Z},(10)$ on p. 430]) gives

$$
\phi\left(P_{1}\right) \approx 0.16728752, \quad \phi\left(P_{2}\right) \approx 0.019066499, \quad \phi\left(P_{3}\right) \approx 0.069180092 .
$$

Using Apecs we computed

$$
\widehat{h}\left(P_{1}\right) \approx 1.6247112, \quad \widehat{h}\left(P_{2}\right) \approx 5.4762626, \quad \widehat{h}\left(P_{3}\right) \approx 2.9083116,
$$

and $\widehat{h}\left(R_{0}\right)=0$.

Then, by the definition of the $A_{i}$ 's, we see that we can take

$$
A_{0}=27.70, \quad A_{1}=A_{2}=A_{3}=19.07, \quad \mathcal{E}=e .
$$

This leads to

$$
c_{4}=2 \cdot 10^{36}\left(\frac{2}{e}\right)^{32} 5^{104} \prod_{i=0}^{3} A_{i}<1.031 \cdot 10^{110} .
$$


With $t_{0}=2, c_{5}=1$, and $c_{6}=1+h_{E}$ in (16), we may choose

$$
c_{7}=2.824, \quad c_{8}=20.73 .
$$

In the notation of (2) and (3), we have

$$
u=1, \quad v=-\frac{337}{3}, \quad w=z=0 .
$$

Moreover,

$$
\gamma=-\frac{2}{3} \cdot 337, \quad \gamma^{\prime}=\frac{1}{3} \cdot 337+337 i, \quad \gamma^{\prime \prime}=\bar{\gamma}^{\prime},
$$

which implies, by Inequality 2 ,

$$
c_{2}=\frac{2}{3} \cdot 337 \sqrt{10}
$$

Further, $c_{1} \approx 0.67736605$, and $c_{3} \approx 5.1930490$, where we have chosen the global minimal Weierstraß model

$$
Y^{2}=X^{3}+X^{2}+75713 X+28375425
$$

for the computation of $c_{3}$. We choose

$$
c_{1}=0.6773, \quad c_{3}=5.194 .
$$

Now (16) immediately implies that

$$
M<4.907 \cdot 10^{59},
$$

provided that $X(P) \geq 711=X_{0}=\left\lfloor\max \left\{c_{2}, u^{2} \gamma+v, v\right\}\right\rfloor+1$. In view of (12), we may take

$$
K_{1}=2318>\frac{4 \sqrt{2}}{\omega} \exp \left(c_{3}\right), \quad K_{2}=0.6773,
$$

and, because of (26),

$$
K_{3}=4.907 \cdot 10^{59} \text {. }
$$

Choosing

$$
K_{0}=10^{245}
$$

which is somewhat larger than $\left(8 \sqrt{6} K_{3}\right)^{4}$, means that we must compute $\phi\left(P_{i}\right)$ for $i=1,2,3$ with a precision of 245 decimal digits. Applying the same implementation of the LLL-algorithm as before, we get a reduced basis $\left\{\mathbf{b}_{1}, \ldots, \mathbf{b}_{\mathbf{4}}\right\}$ with

$\mathbf{b}_{1}=\left(-1.534 \ldots \times 10^{61}, 3.143 \ldots \times 10^{60},-2.629 \ldots \times 10^{60},-3.680 \ldots \times 10^{60}\right)$, from which we see that

$$
\left\|\mathbf{b}_{1}\right\|>1.627 \cdot 10^{61}>8 \sqrt{6} K_{3} .
$$

Therefore, by the Proposition, $M \leq 25$. Repeating the process with $K_{1}, K_{2}$ as above and $K_{3}=25, K_{0}=10^{12}$, the LLL-algorithm gives a reduced basis 
with

$$
\mathbf{b}_{1}=(234,-445,135,-322) \text {. }
$$

Consequently,

$$
\left\|\mathbf{b}_{\mathbf{1}}\right\|=\sqrt{374690}>8 \sqrt{6} K_{3},
$$

so that our Proposition yields the new bound

$$
M \leq 6 .
$$

A direct computer search reveals that the only points

$$
\begin{aligned}
& P=m_{1} P_{1}+m_{2} P_{2}+m_{3} P_{3}+\varepsilon T, \\
& 0 \leq m_{1} \leq 6, \quad-6 \leq m_{2}, m_{3} \leq 6, \quad \varepsilon \in\{0,1\},
\end{aligned}
$$

with integral $X(P) \geq 711$ are those given in the following table:

\begin{tabular}{cccc|cc}
\hline$m_{1}$ & $m_{2}$ & $m_{3}$ & $\varepsilon$ & $X(P)$ & $Y(P)$ \\
\hline 0 & -1 & 0 & 0 & 56784 & -13571615 \\
0 & 1 & 0 & 0 & 56784 & 13571615 \\
2 & 0 & 1 & 1 & 2113 & -105910 \\
\hline
\end{tabular}

A direct search with Apecs for integral points $P$ on $(25)$ with $X(P)<711$ reveals no points other than $(-337,0)$ and $(-287, \pm 3130)$ and this confirms the claim of Example 2.

Appendix: An explicit lower bound for linear forms in elliptic logarithms. We recall the following facts:

- The absolute logarithmic height of $\left(q_{1}, \ldots, q_{n}\right) \in \mathbb{Q}^{n}$ is given by

$$
h\left(q_{1}, \ldots, q_{n}\right)=\sum_{p} \log \max \left\{1,\left|q_{1}\right|_{p}, \ldots,\left|q_{n}\right|_{p}\right\},
$$

where $p$ runs through all primes, including the "infinite" one $\left(|x|_{\infty}=|x|\right.$, the usual absolute value). If $n=1$ and $q_{1}=a / b$ with $\operatorname{gcd}(a, b)=1$, then it is straightforward to check that $h\left(q_{1}\right)=\log \max \{|a|,|b|\}$.

- To any pair of complex numbers $A, B$ such that $\Delta:=A^{3}-27 B^{2} \neq 0$, a so-called Weierstraß $\wp$-function corresponds with invariants $g_{2}=A, g_{3}=B$. This function $\wp$ of a single complex variable is doubly periodic and has one second-order pole in a period parallelogram. Further, $\wp$ satisfies the differential equation $\wp^{\prime}(z)^{2}=4 \wp(z)^{3}-A \wp(z)-B$ and $x=\wp(z), y=$ $\wp^{\prime}(z)$ gives a parameterization over $\mathbb{C}$ of the elliptic curve with Weierstraß equation

$$
y^{2}=4 x^{3}-A x-B=: f_{1}(x),
$$


where $z$ runs through all values of a fundamental parallelogram of the period lattice.

- The fundamental periods $\omega_{1}$ and $\omega_{2}$ of the function $\wp$ may be expressed by the following definite integrals (see [AS, 18.7.4 and 18.7.5 on p. 641]): If $\Delta>0$, then $f_{1}(x)$ has three real zeros $e_{1}>e_{2}>e_{3}$, and

$$
\frac{\omega_{1}}{2}=\int_{e_{1}}^{\infty} \frac{d t}{\sqrt{f_{1}(t)}}, \quad \frac{\omega_{2}}{2}=i \int_{-\infty}^{e_{3}} \frac{d t}{\sqrt{\left|f_{1}(t)\right|}} .
$$

A fast and convenient method for computing the periods numerically is provided by the Arithmetic-Geometric Mean (AGM), see [C]. If the AGM of two positive reals $a$ and $b$ is denoted by $M(a, b)$, then (see [BM, 2.1, in particular (10) and (11)])

$$
\omega_{1}=\frac{\pi}{M\left(\sqrt{e_{1}-e_{3}}, \sqrt{e_{1}-e_{2}}\right)}, \quad \omega_{2}=\frac{\pi i}{M\left(\sqrt{e_{1}-e_{3}}, \sqrt{e_{2}-e_{3}}\right)} .
$$

If $\Delta<0$, then $f_{1}(x)$ has one real root $e_{1}$ and a pair of complex conjugate roots $e_{2}$ and $e_{3}=\bar{e}_{2}$, and

$$
\omega_{1}=\int_{e_{1}}^{\infty} \frac{d t}{\sqrt{f_{1}(t)}}+i \int_{-\infty}^{e_{1}} \frac{d t}{\sqrt{\left|f_{1}(t)\right|}}, \quad \omega_{2}=\bar{\omega}_{1} .
$$

Again, as in the case of a positive discriminant, more convenient formulas exist for the computation of the periods. Consider the curve

$$
Y^{2}=4 X^{3}-4\left(15 e_{1}^{2}-A\right) X-2\left(7 e_{1} A+11 B\right) .
$$

This curve and (27) are 2-isogenous. Consequently, if $\Omega_{1}, \Omega_{2}$ is a pair of fundamental periods for the Weierstraß function associated with (29), then $\omega_{1}=\Omega_{1}+\Omega_{2}, \omega_{2}=\Omega_{1}-\Omega_{2}$ can be taken as a pair of fundamental periods for the Weierstraß function $\wp$ associated with (27). But the right-hand side of (29) has the three real roots

$$
e_{1}+\sqrt{12 e_{1}^{2}-A}>-2 e_{1}>e_{1}-\sqrt{12 e_{1}^{2}-A} .
$$

Therefore, like in the case of a positive discriminant, the periods $\Omega_{1}, \Omega_{2}$ and hence $\omega_{1}, \omega_{2}$ too - can be computed numerically by the AGM method. Finally, the pair of fundamental periods $\omega_{1}, \omega_{2}$ may be chosen such that $\tau=\omega_{2} / \omega_{1}$ satisfies

(30) $\quad|\tau| \geq 1, \Im \tau>0, \quad-\frac{1}{2}<\Re \tau \leq \frac{1}{2} \quad$ with $\Re \tau \geq 0$ if $|\tau|=1$.

Consider the equation

$$
y^{2}=x^{3}+a x+b=: f(x) \quad \text { with } a, b \in \mathbb{Q},
$$

and let $\wp$ be the Weierstraß function corresponding to (31), i.e. the one with invariants $g_{2}=-a / 4, g_{3}=-b / 16$. Note that now $x=4 \wp(z), y=4 \wp^{\prime}(z)$ gives a parameterization over $\mathbb{C}$ of the elliptic curve $E$ defined by (31). 
Matching up the notation of this Appendix with the rest of the paper, we get

$$
f_{1}(x)=4 x^{3}+\frac{a}{4} x+\frac{b}{16}, \quad \gamma=4 e_{1}, \gamma^{\prime}=4 e_{2}, \gamma^{\prime \prime}=4 e_{3},
$$

where $\gamma$ is the largest (or the only) real zero, and $\gamma^{\prime}, \gamma^{\prime \prime}$ are the remaining zeros of $f(x)$. In view of the foregoing discussion on the periods, it immediately follows that a pair of fundamental periods is given by

$$
\omega_{1}=\frac{2 \pi}{M\left(\sqrt{\gamma-\gamma^{\prime \prime}}, \sqrt{\gamma-\gamma^{\prime}}\right)}, \quad \omega_{2}=\frac{2 \pi i}{M\left(\sqrt{\gamma-\gamma^{\prime \prime}}, \sqrt{\gamma^{\prime}-\gamma^{\prime \prime}}\right)}
$$

in the case of three real roots $\gamma>\gamma^{\prime}>\gamma^{\prime \prime}$, and by

$$
\omega_{1}=\Omega_{1}+\Omega_{2}, \quad \omega_{2}=\Omega_{1}-\Omega_{2},
$$

with

$$
\begin{aligned}
& \Omega_{1}=\frac{\pi}{M\left(\sqrt[4]{3 \gamma^{2}+a}, \frac{1}{2} \sqrt{3 \gamma+2 \sqrt{3 \gamma^{2}+a}}\right)}, \\
& \Omega_{2}=\frac{\pi i}{M\left(\sqrt[4]{3 \gamma^{2}+a}, \frac{1}{2} \sqrt{-3 \gamma+2 \sqrt{3 \gamma^{2}+a}}\right)},
\end{aligned}
$$

in the case of a single real root $\gamma$.

Let $u_{0}, \ldots, u_{r} \in \mathbb{C}$ be such that, for every $i=0, \ldots, r, R_{i}=$ $\left(4 \wp\left(u_{i}\right), 4 \wp^{\prime}\left(u_{i}\right)\right) \in E(\mathbb{Q}) \cup\{O\}$ on $(31)$-note that $R_{i}=O$ means that $u_{i}$ is a pole of $\wp$.

Let $j_{E}=2^{8} 3^{3} a^{3} /\left(4 a^{3}+27 b^{2}\right)$ be the $j$-invariant of $E$ and define

$$
h_{E}=\max \left\{1, h(a / 4, b / 16), h\left(j_{E}\right)\right\} .
$$

Let $\omega_{1}, \omega_{2}$ be a pair of fundamental periods for $\wp$ with $\tau=\omega_{2} / \omega_{1}$ satisfying (30). For every $i=0, \ldots, r$, consider a positive number $A_{i}$ such that

$$
A_{i} \geq \max \left\{\widehat{h}\left(R_{i}\right), h_{E}, \frac{3 \pi u_{i}^{2}}{\left|\omega_{1}\right|^{2} \Im \tau}\right\},
$$

where $\widehat{h}$ denotes the usual Néron-Tate or canonical height function. Further, consider a number $\mathcal{E}$ satisfying

$$
e \leq \mathcal{E} \leq \min _{i=0, \ldots, r}\left\{\frac{e\left|\omega_{1}\right| \sqrt{A_{i} \Im \tau}}{\left|u_{i}\right| \sqrt{3 \pi}}\right\}
$$

and finally, let $L$ be the linear form

$$
L=\frac{b_{0}}{t} u_{0}+b_{1} u_{1}+\ldots+b_{r} u_{r}
$$

where $t, b_{0}, b_{1}, \ldots, b_{r} \in \mathbb{Z}$ and $t>0$. Also let $B$ be a positive integer such that

$$
B \geq \max \left\{A_{0}, \ldots, A_{r}, t,\left|b_{0}\right|,\left|b_{1}\right|, \ldots,\left|b_{r}\right|, 16\right\} .
$$


The following theorem is a special case of [D, Théorème 2.1].

DAVID's Theorem. If $L \neq 0$, then

$$
|L| \geq \exp \left(-c_{4}(\log B+\log \mathcal{E})\left(\log \log B+\log \mathcal{E}+h_{E}\right)^{r+2}\right),
$$

where

$$
c_{4}=2 \cdot 10^{7 r+15}\left(\frac{2}{e}\right)^{2(r+1)^{2}}(r+2)^{4 r^{2}+18 r+14}(\log \mathcal{E})^{-2 r-3} \prod_{i=0}^{r} A_{i} .
$$

\section{References}

[AS] M. Abramowitz and I. Stegun (eds.), Handbook of Mathematical Functions, Dover, New York, 1964.

[BM] J.-B. Bost et J.-F. Mestre, Moyenne arithmético-géométrique et périodes des courbes de genre 1 et 2, Gazette de Mathématiciens, S.M.F., Octobre 1988.

[Br] A. Bremner, An equation of Mordell, Math. Comp. 29 (1975), 925-928.

[C] D. A. Cox, The arithmetic-geometric mean of Gauss, Enseign. Math. 30 (1984), 275-330.

[D] S. David, Minorations de formes linéaires de logarithmes elliptiques, Publ. Math. Univ. Pierre et Marie Curie 106, Problèmes diophantiens 1991-1992, exposé no. 3.

[H] N. Hirata-Kohno, Formes linéaires de logarithmes de points algébriques sur les groupes algébriques, Invent. Math. 104 (1991), 401-433.

[La] S. Lang, Elliptic Curves; Diophantine Analysis, Grundlehren Math. Wiss. 231, Springer, Berlin, 1978.

[LLL] A. K. Lenstra, H. W. Lenstra Jr., and L. Lovász, Factoring polynomials with rational coefficients, Math. Ann. 261 (1982), 515-534.

[Lj] W. Ljunggren, A diophantine problem, J. London Math. Soc. (2) 3 (1971), 385-391.

[Ma] D. W. Masser, Elliptic Functions and Transcendence, Lecture Notes in Math. 437, Springer, Berlin, 1975.

[Mo] L. J. Mordell, Diophantine Equations, Pure Appl. Math. 30, Academic Press, London and New York, 1969.

[S1] J. H. Silverman, The Arithmetic of Elliptic Curves, Graduate Texts in Math. 106, Springer, New York, 1986.

[S2] —, Computing heights on elliptic curves, Math. Comp. 51 (1988), 339-358.

[S3] -, The difference between the Weil height and the canonical height on elliptic curves, ibid. 55 (1990), 723-743.

[STo] R. J. Stroeker and J. Top, On the equation $Y^{2}=(X+p)\left(X^{2}+p^{2}\right)$, Rocky Mountain J. Math. 24 (2) (1994), to appear.

[STz] R. J. Stroeker and N. Tzanakis, On the application of Skolem's p-adic method to the solution of Thue equations, J. Number Theory 29 (2) (1988), 166-195.

[TdW] N. Tzanakis and B. M. M. de Weger, On the practical solution of the Thue equation, ibid. 31 (2) (1989), 99-132. 
[dW] B. M. M. de Weger, Algorithms for Diophantine Equations, CWI Tract 65, Stichting Mathematisch Centrum, Amsterdam, 1989.

[WW] E. T. Whittaker and G. N. Watson, A Course of Modern Analysis, 4th ed., Cambridge University Press, New York, 1978.

[Wu G. Wüstholz, Recent progress in transcendence theory, in: Number Theory, Noordwijkerhout 1983, Lecture Notes in Math. 1068, Springer, Berlin, 1984, 280-296.

[Z] D. Zagier, Large integral points on elliptic curves, Math. Comp. 48 (1987), 425-436.

ECONOMETRIC INSTITUTE

ERASMUS UNIVERSITY ROTTERDAM

P.O. BOX 1738

3000 DR ROTTERDAM, THE NETHERLANDS

E-mail: STROEKER@WIS.FEW.EUR.NL
DEPARTMENT OF MATHEMATICS

UNIVERSITY OF CRETE

P.O. BOX 470

71409 IRAKLION, GREECE

E-mail: TZANAKIS@TALOS.CC.UCH.GR 